\title{
Systematic review of general burden of disease studies using disability-adjusted life years
}

\author{
Suzanne Polinder ${ }^{1 *}$, Juanita A Haagsma ${ }^{1}$, Claudia Stein ${ }^{2}$ and Arie H Havelaar ${ }^{3,4}$
}

\begin{abstract}
Objective: To systematically review the methodology of general burden of disease studies. Three key questions were addressed: 1) what was the quality of the data, 2) which methodological choices were made to calculate disability adjusted life years (DALYs), and 3) were uncertainty and risk factor analyses performed? Furthermore, DALY outcomes of the included studies were compared.

Methods: Burden of disease studies (1990 to 2011) in international peer-reviewed journals and in grey literature were identified with main inclusion criteria being multiple-cause studies that quantified the burden of disease as the sum of the burden of all distinct diseases expressed in DALYs. Electronic database searches included Medline (PubMed), EMBASE, and Web of Science. Studies were collated by study population, design, methods used to measure mortality and morbidity, risk factor analyses, and evaluation of results.

Results: Thirty-one studies met the inclusion criteria of our review. Overall, studies followed the Global Burden of Disease (GBD) approach. However, considerable variation existed in disability weights, discounting, age-weighting, and adjustments for uncertainty. Few studies reported whether mortality data were corrected for missing data or underreporting. Comparison with the GBD DALY outcomes by country revealed that for some studies DALY estimates were of similar magnitude; others reported DALY estimates that were two times higher or lower.
\end{abstract}

Conclusions: Overcoming "error" variation due to the use of different methodologies and low-quality data is a critical priority for advancing burden of disease studies. This can enlarge the detection of true variation in DALY outcomes between populations or over time.

Keywords: Review, General burden of disease, Disability-adjusted life years, Methodology

\section{Introduction}

The burden of disease concept provides a conceptual and methodological framework to quantify and compare the health of populations using a summary measure of both mortality and disability - the disability-adjusted life year (DALY) [1,2]. Since the launch of the Global Burden of Disease (GBD) study in 1993, the burden of disease concept has been widely adopted by countries and health development agencies alike to identify the relative magnitude of different health problems. This information serves as crucial input for debates about priorities in the health sector.

Criticism of the GBD study focused on the construction of DALYs [3,4], particularly the social choices

\footnotetext{
* Correspondence: s.polinder@erasmusmc.nl

'Department of Public Health, Erasmus MC, Rotterdam, Netherlands

Full list of author information is available at the end of the article
}

around age weights and severity scores of disabilities. The GBD 2010 Study that is currently being conducted responded to the critiques and recent improvements in the field and includes significantly improved methods for burden assessment, particularly for ranking risk factors and disabilities [5,6]. It is expected that the imminent publication of the GBD 2010 Study will result in a new impulse to perform burden of disease studies.

A major strength of the burden of disease concept is that it allows comparison between different health problems, between different years, and between countries. In principle, the DALY approach should be used consistently to provide comparable DALY estimates. However, the technical approach of the GBD is complex, both in concept and in application, and there are many methodological alternatives, e.g., using alternative morbidity estimates, life expectancies, or severity weights, which

\section{Biomed Central}

(c) 2012 Polinder et al.; licensee BioMed Central Ltd. This is an Open Access article distributed under the terms of the Creative Commons Attribution License (http://creativecommons.org/licenses/by/2.0), which permits unrestricted use, distribution, and reproduction in any medium, provided the original work is properly cited. 
have enormous influence on DALY outcomes [7]. Hence, the interpretation of results of burden of disease studies requires detailed methodological knowledge.

General burden of disease studies are multiple-cause studies that quantify the burden of disease as the sum of the burden of all distinct diseases expressed in DALYs. Until now, a systematic review of general burden of disease studies and the underlying methodological choices has not been conducted. This review was a first step in the development of a protocol specifically for burden of foodborne disease studies. This protocol complements the GBD manual, as it addresses problems that arise particularly when undertaking foodborne burden of disease studies. The protocol was developed for researchers that aim to undertake burden of foodborne disease studies in the framework of the Foodborne Disease Burden Epidemiology Reference Group (FERG). The FERG was established in 2007 by the World Health Organization (WHO). The purpose of the group is to advise WHO in their estimates of the global burden of diseases commonly transmitted through food.

This systematic review aims to provide an overview of the methodology of general burden of disease studies using the DALY approach. In the review, the following key questions were addressed: 1) what was the quality of the data and were there any data gaps, 2) which methodological choices were made in order to calculate years of life lost due to mortality (YLL) and years lost due to disability (YLD), and 3) which methods were used to handle uncertainty and risk factor analysis. Furthermore, DALY outcomes for specific disease and injury groups resulting from the general burden of disease studies were compared.

\section{Methods}

\section{Selection criteria}

In this review, burden of disease studies based on general multiple-cause studies (including all diseases and injuries) were included. Empirical studies in international peer-reviewed journals and grey literature published in English in the period 1990 to 2011 were included. Studies in established market economies and low- and middle-income countries were also included. The review is restricted to studies using the DALY as a burden of disease measure, both country-specific and worldwide.

\section{Disability-adjusted life year}

The DALY is calculated by adding YLL to morbidity and disability, expressed in YLD. The DALY methodology is represented in a conceptual framework in Figure 1.

YLL is calculated by summation of the number of fatal cases (d) due to health outcome (x) in a certain period multiplied by the residual expected life expectancy (e) at the age of death:

$$
\mathrm{YLL}_{\mathrm{x}}=\Sigma \mathrm{d}_{\mathrm{x}} \times \mathrm{e}_{\mathrm{x}}
$$

For the calculation of YLD an incidence or prevalencebased approach can be used, which is highly dependent on the availability of data. The incidence-based approach quantifies both the burden of disease occurring during the reference period and the burden accrued into the future. A prevalence-based approach ascribes burden to the age at which disability is lived.

$\mathrm{YLD}_{\text {inc }}$ is calculated by multiplying the number of incident cases (I) at a certain age with health outcome $(x)$ by the duration of the health outcome $(\mathrm{t})$ and the disability weight $(\mathrm{dw})$ assigned to health outcome $\mathrm{x}$ :

$$
\mathrm{YLD}_{\text {incx }}=\Sigma \mathrm{I}_{\mathrm{x}} \times \mathrm{t}_{\mathrm{x}} \times \mathrm{dw}_{\mathrm{x}}
$$

$\mathrm{YLD}_{\text {prev }}$ is calculated by multiplying the number of prevalent cases $(\mathrm{P})$ in age group $(\mathrm{x})$ at a point in the reference period with the disability weight $(\mathrm{dw})$ assigned to health outcome $\mathrm{x}: \mathrm{YLD}_{\text {prev } \mathrm{x}}=\mathrm{P}_{\mathrm{x}} \times \mathrm{dw}_{\mathrm{x}}$ These basic formulas can be supplemented due to methodological choices (e.g., expanding with discount factor and ageweighting).

\section{Data sources and search strategy}

Searches of eligible studies were conducted in Medline (PubMed), EMBASE, and Web of Science. Searches for eligible grey literature were conducted in Google Scholar and SIGLE (System for Information on Grey Literature in Europe). All international peer-reviewed articles and grey literature published in English in the period January 1990 to 2011 were included in the searches. Search terms used for general burden of disease studies were: "burden of disease, "disability adjusted life year," "disabilityadjusted life year," "DALY." Keywords were matched to database-specific indexing terms. In addition to database searches, reference lists of review studies and articles included in the review were screened for titles that included key terms.

\section{Data extraction}

Relevant papers were selected by screening the titles (first step), abstracts (second step), and entire articles (third step) retrieved through the database searches. During each step respectively, the title, abstract, or entire article was screened to ensure that it met the selection criteria listed above. This screening was conducted independently by two researchers (Suzanne Polinder and Juanita Haagsma). Disagreement about eligibility between the reviewers was solved through discussion.

Full articles were critically appraised by two reviewers (Suzanne Polinder and Juanita Haagsma), using data 


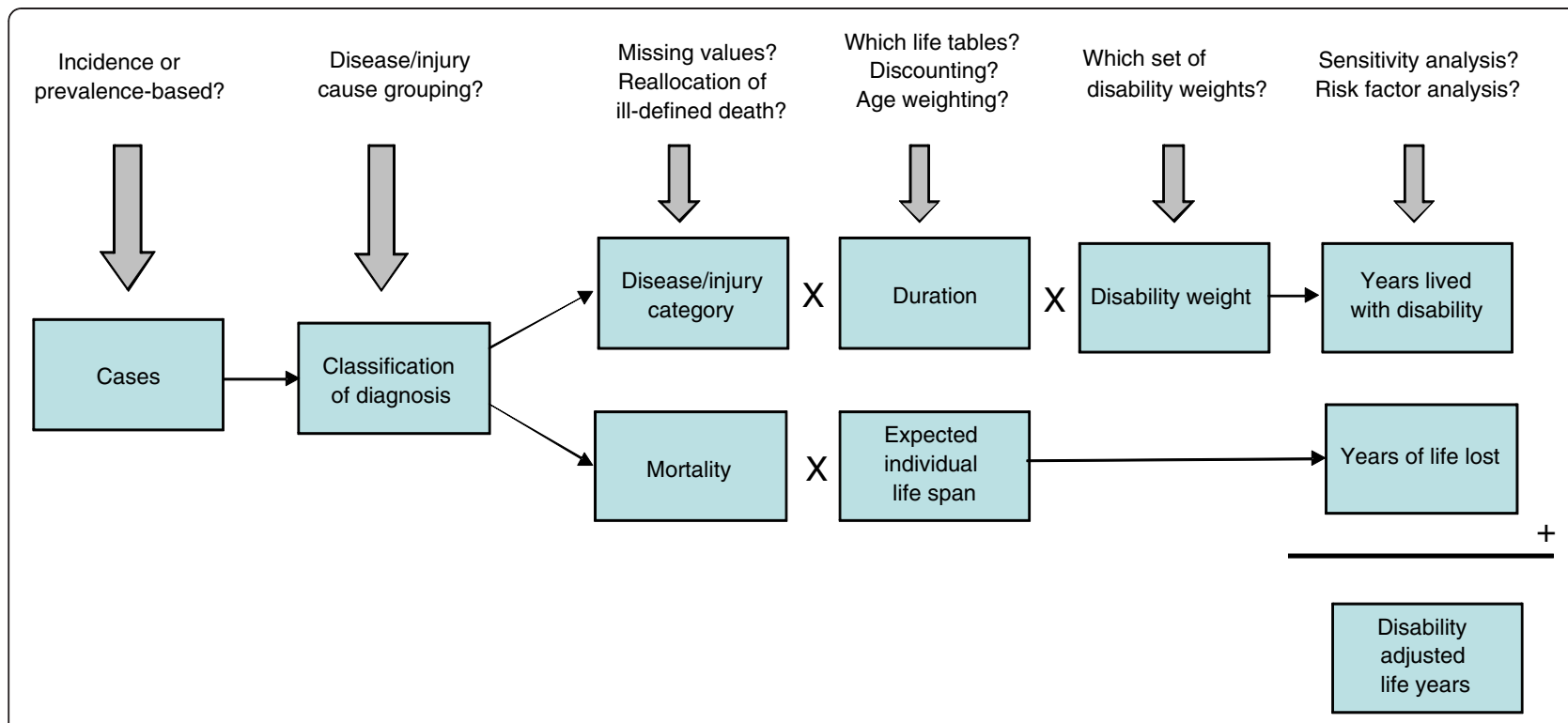

Figure 1 Conceptual model.

extraction forms, which included information on the study population, details regarding the methods used to calculate YLL and YLD, risk factor analysis, main conclusions, and evaluation of results. Their reports were compared and disagreements were resolved by discussion.

\section{Results}

\section{Literature search}

Figure 2 shows the flow diagram of the search of existing burden of disease studies and main reasons for exclusion. Eventually, 31 studies were included in the review. Table 1 shows the studies that have been included for the review. In Figure 3, the number of general burden of disease studies is shown per WHO region. Four studies were worldwide burden of disease studies [8-11].

\section{What was the quality of the data and were there any data gaps?}

The availability and quality of mortality and morbidity data strongly differs by country.

Most countries register the number of fatal cases as well as the age and cause of death in national vital registrations (see Table 1). Vital registration often had full coverage, which means that the data are representative for the population of these countries.

Where vital registration had less than $100 \%$ coverage it is important to know whether the data had been extrapolated to $100 \%$. However, the majority of the studies did not report whether the death statistics used had $100 \%$ coverage. Only 10 studies reported that they corrected for underreporting of death statistics [8,10-18], for instance with demographic projection models [14], or by using the average of several years of death statistics to minimize stochastic variation $[10,18]$.

Next to extrapolation in case of missing data, procedures such as reallocating of ill-defined deaths from so-called "garbage codes" may be necessary. Problems can arise from the routine use of specific codes in the International Classification of Diseases (ICD) list where information is incomplete. This can occur when medical records are not fully considered or when medical practitioners concerned with the specific cases are not consulted during the process of completing the forms. Certain codes then become overused and bias the relative importance attached to particular cases of death. These codes are called "garbage codes." The majority of the studies ( $\mathrm{n}=24$; Table 2) reported corrections for ill-defined deaths, partly by reallocation from garbage codes. For instance, the Victorian Burden of Disease study [19] redistributed the cardiovascular garbage codes to ischemic heart disease, inflammatory heart disease, and hypertensive heart disease in proportions that varied by age. Notably, many studies did not report whether mortality data were corrected for missing data, underreporting, or misclassification.

\section{Which methodological choices were made in order to calculate YLL and YLD General burden of disease methods}

An incidence- or prevalence-based method can be used to quantify the burden of disease. In practice, it is often difficult to rigidly apply the incidence or prevalencebased approach and sometimes compromises must be made. Most studies have followed an incidence-based 


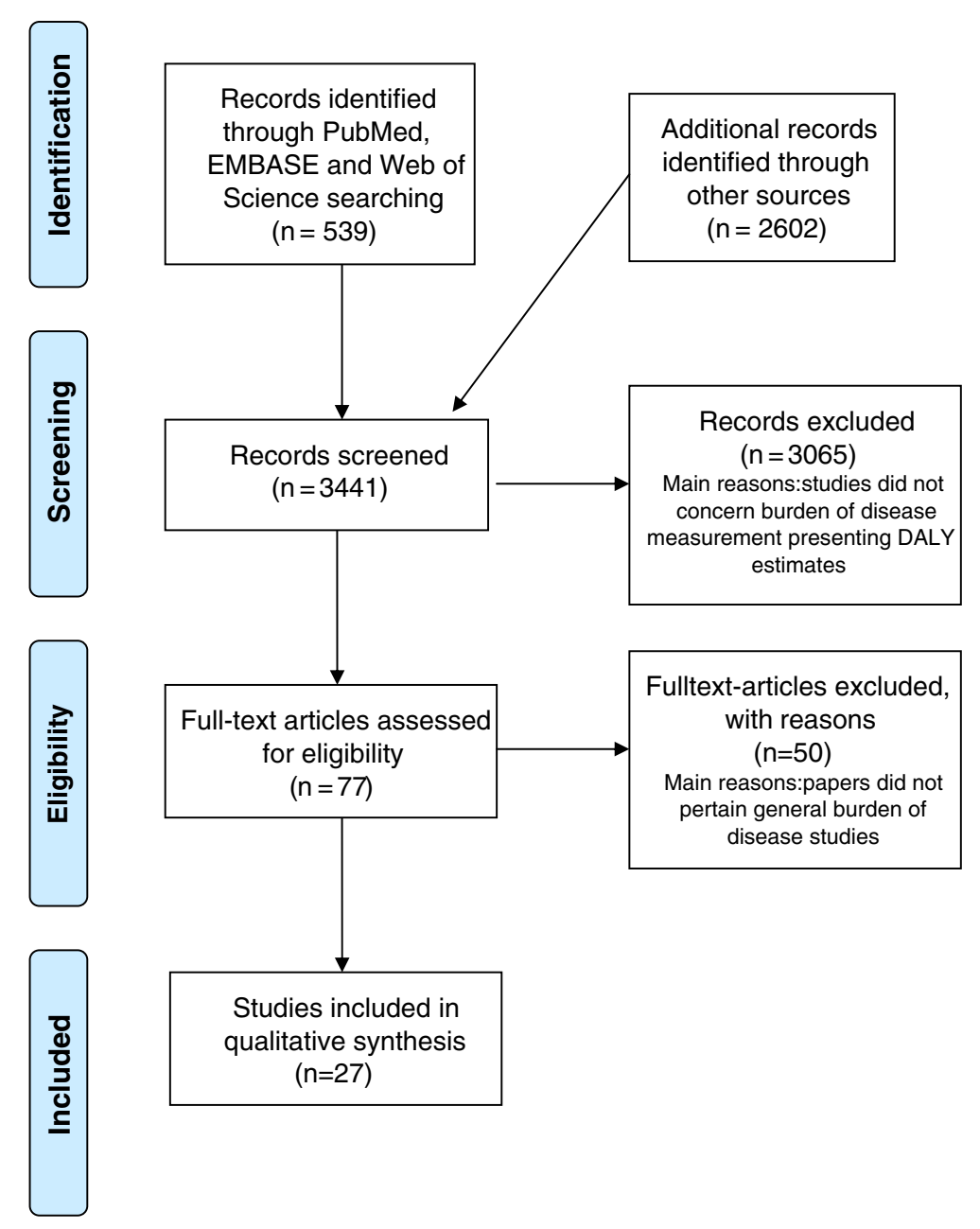

Figure 2 Flow diagram of the search of existing burden of disease studies.

approach $(n=25$, Table 2). For some of these countries, not all incidence-based data could be gathered, and partly the prevalence-based method was used.

The GBD developed a list of disease and injury causes based on the ICD. Including all causes avoids the problems of overinclusiveness of single-cause studies and incompatible mortality claims for different causes. Most studies used the GBD disease and injury causes, and sometimes some causes were removed due to little relevance and other causes were added to the list (Table 2). Three studies developed their own disease and injury groups [20-22]. The original GBD study applied age-weighting and discounting [23]. With age-weighting, the altering levels of dependency with age are taken into account, meaning that years lived at youngest and oldest age are given less weight. Discounting means that future life years are assigned less value than those lived today. This is based on the economic concept that immediate profits are generally preferred over benefits later in time [1]. Both age-weighting and discounting have been disputed, which is further described in the discussion section. This debate is also translated in the distinction in the use of both methodologies in the included studies. Almost all studies $(n=26)$ assigned less value to future life years by using a discount factor. However, only half of the studies $(n=17)$ performed ageweighting in their study. It was not always stated whether age-weighting and discounting were used [18,24].

\section{Methods to calculate YLL}

Country-specific or model life tables with life expectancy data can be used to calculate YLLs. Most studies used the Coale and Demeny West Level 26 and 25 life tables, developed by GBD ( $n=23$; Table 2). The West Level 26 and 25 life tables are global model life tables that have a standard life expectancy at birth: 80.0 years for males and 82.5 years for females [25]. Other studies used life tables from their country $(n=8)[18,21,26-31]$. 


\begin{tabular}{|c|c|c|c|c|}
\hline Author, Year (reference no.) & Country $^{1}$ & WHO region & Data sources mortality & Data sources morbidity \\
\hline Begg, 2008 [13] & Australia & WPRO & Australian death registry from Australian Bureau of Statistics & $\begin{array}{l}\text { Disease registers, cohort and intervention studies } \\
\text { and surveys }\end{array}$ \\
\hline Bowie, 1997 [22] & $\begin{array}{l}\text { South and West region, } \\
\text { England }\end{array}$ & EURO & $\begin{array}{l}1992 \text { Office of Population Censuses and Surveys age-specific } \\
\text { mortality data }\end{array}$ & World bank study \\
\hline Bradshaw, 2003 [14] & South Africa & AFRO & $\begin{array}{l}\text { 1) Statistics South Africa, 2) Population Register, 3) National } \\
\text { Injury Mortality Surveillance Study, 4) For children: } \\
1996 \text { census and the } 1998 \text { Demographic and Health Survey }\end{array}$ & The South African Birth Defects Surveillance System \\
\hline Bundhamchareon, 2002 [16] & Thailand & SEARO & Survey of Population Change & $\begin{array}{l}\text { Disease registers, routine databases or epidemiological } \\
\text { studies }\end{array}$ \\
\hline Chapman, 2006 [41] & Zimbabwe & AFRO & $\begin{array}{l}\text { Vital Registration System and number of deaths for } 1997 \\
\text { taken from a nationwide census }\end{array}$ & $\begin{array}{l}\text { Local disease registers, surveys and routine health } \\
\text { service data supplemented by estimates from } \\
\text { epidemiological studies from other settings }\end{array}$ \\
\hline Dodhia, 2008 [50] & London, England & EURO & Local mortality data & $\begin{array}{l}\text { The GBD estimates from World Health Organization } \\
\text { were used }\end{array}$ \\
\hline Hyder, 2000 [40] & Pakistan & EMRO & $\begin{array}{l}\text { Pakistan Demographic Survey of 1989, Pakistan Demographic } \\
\text { and Health Survey 1990-1991, National Health Survey of } \\
\text { Pakistan 1989-1994 }\end{array}$ & $\begin{array}{l}\text { More than } 180 \text { national and local data sources were } \\
\text { reviewed to obtain information on diseases in Pakistan }\end{array}$ \\
\hline Innove Solutions, 1998 [51] & West Pennine, England & EURO & National mortality data from Public Health Department & West Pennine Health Authority morbidity data \\
\hline Jankovic, $2007[52]$ & $\begin{array}{l}\text { Serbia and Serbia } \\
\text { Montenegro }\end{array}$ & EURO & Serbian Office of Statistics mortality database & $\begin{array}{l}\text { Disease registers, routine databases, and epidemiological } \\
\text { studies }\end{array}$ \\
\hline Laaser, 2007 [38] & Syria & EMRO & $\begin{array}{l}\text { National mortality register, analysis of the WHO life table } \\
\text { of Syria and mortality sentinel surveillance }\end{array}$ & National databases \\
\hline Lai, 2009 [27] & Estonia & EURO & Vital registration of Statistics Estonia & Estonian Health Insurance Fund database \\
\hline Lopez, 2006 [9] & Global & Global & $\begin{array}{l}\text { Death registrations, population-based epidemiological } \\
\text { studies, disease registers, and notification systems. }\end{array}$ & $\begin{array}{l}\text { Disease registers, epidemiological studies, health surveys, } \\
\text { and health facility data }\end{array}$ \\
\hline Mathers, 2001 [28] & Australia & WPRO & Australian death registry from Australian Bureau of Statistics & Disease registers and epidemiological studies \\
\hline Melse, 2000 [21] & Netherlands & & Dutch death registration & $\begin{array}{l}\text { General practitioner registrations, national registries, } \\
\text { and population surveys }\end{array}$ \\
\hline Michaud, 2006 [35] & United States & AMRO & $\begin{array}{l}\text { Mortality File from the National Center for Health } \\
\text { Statistics } 1996\end{array}$ & $\begin{array}{l}\text { National health surveys, the National Hospital Discharge } \\
\text { Database, disease registers, and epidemiological studies }\end{array}$ \\
\hline Murray, $1997[10]$ & Global & $\begin{array}{l}21 \text { regions in } \\
\text { the world }\end{array}$ & $\begin{array}{l}\text { Death registration systems, sample death registration systems, } \\
\text { epidemiological assessments, cause of death models }\end{array}$ & $\begin{array}{l}\text { Disease registers, population surveys, epidemiological } \\
\text { studies, health facility data }\end{array}$ \\
\hline Naghavi, 2009 [31] & Iran & EMRO & data from the national death registry of Health Ministry ME & $\begin{array}{l}\text { Disease surveillance systems, hospital disease registries, } \\
\text { representative national surveys, subnational and } \\
\text { local studies. }\end{array}$ \\
\hline Phua, 2009 [39] & Singapore, Malaysia & WPRO & Registry of Births and Deaths & $\begin{array}{l}\text { National disease registers and surveillance or notification } \\
\text { systems, national health surveys, health services } \\
\text { utilization data }\end{array}$ \\
\hline Pike, 2002 [30] & Queensland, Australia & WPRO & Australian death registry from Australian Bureau of Statistics & Australian BoD YLD data \\
\hline
\end{tabular}


Table 1 Study characteristics of included studies (Continued)

\begin{tabular}{|c|c|c|c|c|}
\hline $\begin{array}{l}\text { SA Department of Health } \\
\text { Australia, } 2005 \text { [12] }\end{array}$ & South Australia & WPRO & Australian death registry from Australian Bureau of Statistics & Disease registers and epidemiological studies \\
\hline Somerford, 2004 [29] & Western Australia & WPRO & Australian death registry from Australian Bureau of Statistics & Australian BoD YLD data \\
\hline Stevens, 2008 [26] & Mexico & AMRO & National Mortality statistics & $\begin{array}{l}\text { Vital statistics, national censuses, health examination } \\
\text { surveys, and published epidemiological studies }\end{array}$ \\
\hline Tobias, 2001 [20] & New Zealand & WPRO & New Zealand Health Information Service mortality & $\begin{array}{l}\text { Disease registers, population surveys, hospital } \\
\text { discharge register }\end{array}$ \\
\hline Ünüvar, 2004 [15] & Turkey & EURO & $\begin{array}{l}\text { Death statistics obtained from provincial and town centers } \\
\text { in Turkey, hospital records and Directorate General of } \\
\text { Security data }\end{array}$ & $\begin{array}{l}\text { Population Census, Records obtained from government } \\
\text { agencies, National surveys, national and international } \\
\text { reports and articles }\end{array}$ \\
\hline Victorian BOD, 2005 [19] & Victoria, Australia & WPRO & Australian death registry from Australian Bureau of Statistics & $\begin{array}{l}\text { Disease registers, routine databases and population } \\
\text { health surveys }\end{array}$ \\
\hline $\begin{array}{l}\text { World Health Organization, } \\
2008[8]\end{array}$ & Global & $\begin{array}{l}21 \text { regions in } \\
\text { the world }\end{array}$ & $\begin{array}{l}\text { Death registration systems, sample death registration systems, } \\
\text { epidemiological assessments, cause of death models }\end{array}$ & $\begin{array}{l}\text { Disease registers, population surveys, epidemiological } \\
\text { studies, health facility data }\end{array}$ \\
\hline $\begin{array}{l}\text { World Health Organization, } \\
2009 \text { [11] }\end{array}$ & Global & $\begin{array}{l}21 \text { regions in } \\
\text { the world }\end{array}$ & $\begin{array}{l}\text { Death registration systems, sample death registration systems, } \\
\text { epidemiological assessments, cause of death models }\end{array}$ & $\begin{array}{l}\text { Disease registers, population surveys, epidemiological } \\
\text { studies, health facility data }\end{array}$ \\
\hline Yoon, 2007 [24] & Korea & SEARO & The Korean National Health Insurance system & Large normative cohort \\
\hline Yusoff, 2004 [17] & Malaysia & WPRO & $\begin{array}{l}\text { Malaysian mortality data of the Department of Statistics } \\
\text { subdivided in four regions }\end{array}$ & $\begin{array}{l}\text { Disease registries, routine databases and epidemiological } \\
\text { studies }\end{array}$ \\
\hline Zhao, 2004 [18] & $\begin{array}{l}\text { Northern Territory, } \\
\text { Australia }\end{array}$ & WPRO & Australian death registry from Australian Bureau of Statistics & Disease registers, population surveys, expert opinions \\
\hline Zhou, 2011 [36] & $\begin{array}{l}\text { Yunnan Province, } \\
\text { China }\end{array}$ & WPRO & $\begin{array}{l}\text { Medical death certificate information from Centers for Disease } \\
\text { Control and Prevention }\end{array}$ & $\begin{array}{l}\text { Indirect method: YLD/YLL ratio for China taken from the } \\
\text { WHO World Health Report } 2002\end{array}$ \\
\hline
\end{tabular}

Country / region and country of the study population. 


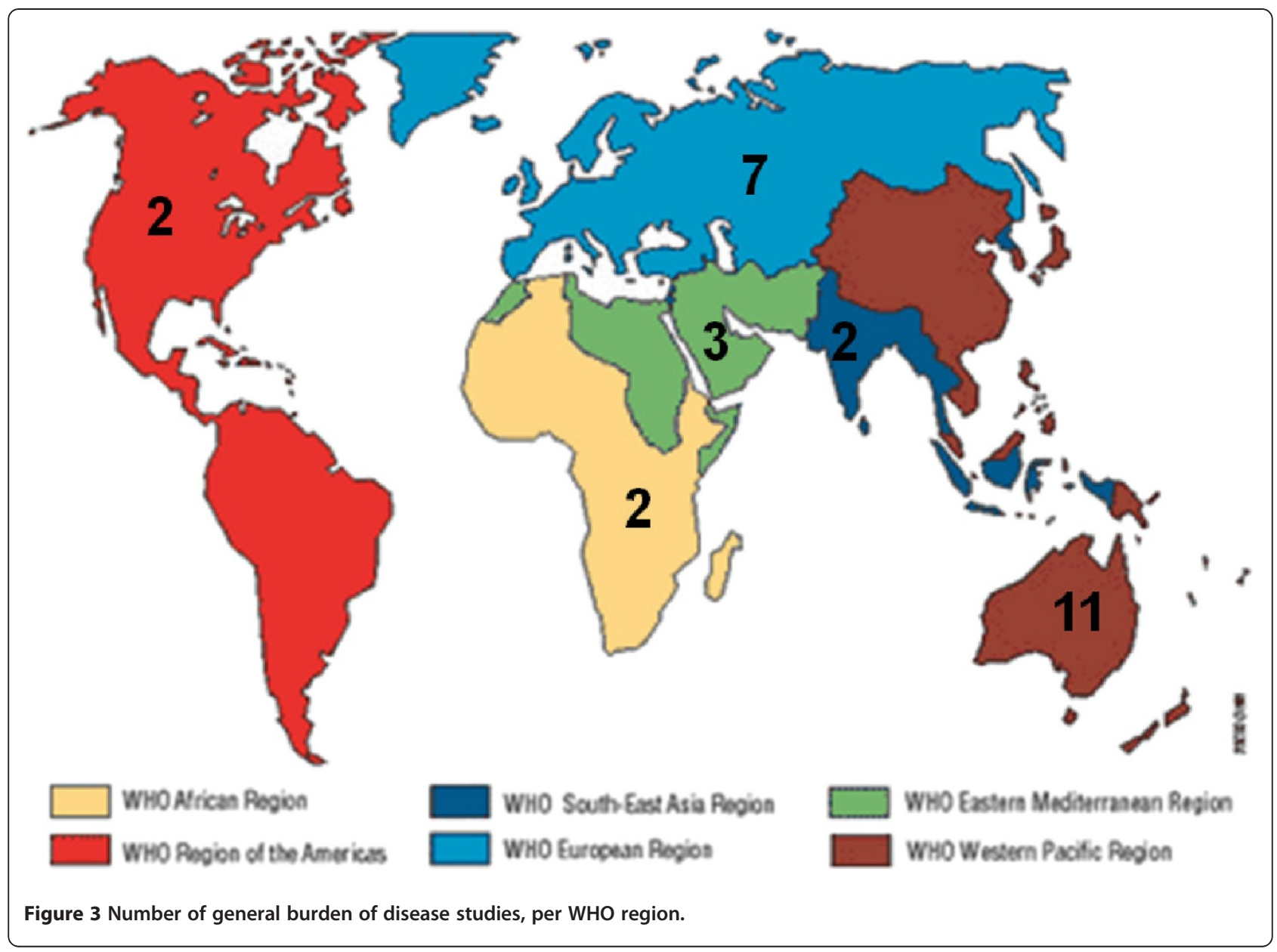

Methods to calculate years lost due to disability (YLD)

A crucial aspect to calculating YLD is the disability weight; a value ranging from 1 , indicating worst imaginable health state equal to death, through 0 , indicating full health. Its value is based on the preferences stated by a panel of judges towards a set of hypothetical health states, expressing the relative undesirability of the health state [32,33]. Several sets of disability weights exist, such as the GBD disability weights [1] and the Dutch Disability Weights (DDW) [34]. Most studies ( $\mathrm{n}=27$; Table 2) used the GBD disability weights. Sixteen of these studies combined the GBD weights with the DDW for disease and injury causes that were not in the GBD study.

Four studies $[14,29,35,36]$ derived YLD by applying the ratio of YLD to YLL from one study to derive the YLDs for their own study, which is common in burden of disease analyses for countries with limited data on disease occurrence [25]. For instance, the Western Australian Burden of Disease study [29] used this method to derive the YLDs for residual conditions not specifically analysed, but which were grouped to complete a broad disease grouping (e.g., other cardiovascular conditions).
Eight studies adjusted for comorbidity [12,13,16,19,20, 28,30,31]. The Australian burden of disease studies $[13,19,28,30]$ have developed methods to address the issue of comorbidity for the common coexisting nonfatal conditions (e.g., deafness, osteoarthritis, mental retardation, diabetes). With this method, the difference between a composite weight for two coexisting conditions and the weight for the more severe of the conditions is calculated and used, rather than the weight of the milder condition in its independent state. The disability weight for the more severe condition remains unchanged.

Which methods were used to handle uncertainty and risk factor analysis?

\section{Uncertainty analysis}

Each burden of disease study contains uncertainty as a result of possible imprecision in epidemiological data (e.g., deaths, incidence, prevalence, severity), in the parameter values used or due to methodological controversy. None of the studies quantify uncertainty in epidemiological data. Uncertainty in the parameter values is 
Table 2 Methods used to calculate YLL and YLD in general burden of disease studies

\begin{tabular}{|c|c|c|c|c|c|c|c|c|c|c|c|c|}
\hline \multirow{2}{*}{$\begin{array}{l}\text { Author, Year } \\
\text { (reference no.) }\end{array}$} & \multicolumn{7}{|c|}{ General burden of disease methods } & \multicolumn{2}{|l|}{ YLL methodology } & \multicolumn{3}{|c|}{ YLD methodology } \\
\hline & $\begin{array}{l}\text { Incidence or } \\
\text { prevalence } \\
\text { based } \\
\text { approach }\end{array}$ & $\begin{array}{l}\text { Disease and } \\
\text { injury cause } \\
\text { groups used }\end{array}$ & $\begin{array}{l}\text { Were } \\
\text { missing } \\
\text { values } \\
\text { handled? }\end{array}$ & Discounting & $\begin{array}{l}\text { Age } \\
\text { weighting }\end{array}$ & $\begin{array}{l}\text { Sensitivity } \\
\text { analysis }\end{array}$ & $\begin{array}{l}\text { Risk } \\
\text { factor } \\
\text { analysis }\end{array}$ & $\begin{array}{l}\text { Which life } \\
\text { tables used? }\end{array}$ & $\begin{array}{l}\text { Reallocation } \\
\text { of ill-defined } \\
\text { death? }(y / n)\end{array}$ & $\begin{array}{l}\text { Disability } \\
\text { weights }\end{array}$ & $\begin{array}{l}\text { Distribution } \\
\text { by severity? }\end{array}$ & $\begin{array}{l}\text { Adjustment } \\
\text { for comorbidity }\end{array}$ \\
\hline GBD approach & Incidence & GBD list I, II, III & $\mathrm{Y}$ & $\mathrm{Y}$ & $\mathrm{Y}$ & $\mathrm{Y}$ & $\mathrm{Y}$ & $\begin{array}{l}\text { Standard West } \\
25 \text { (males) and } \\
26 \text { (females) }\end{array}$ & $\mathrm{Y}$ & GBD & $\mathrm{N}$ & $\mathbf{N}$ \\
\hline Begg, 2008 [13] & Incidence & GBD list I, II, III & Y & Y & Y & Y & Y & $\begin{array}{l}\text { Standard West } \\
25 \text { (females) and } \\
26 \text { (males) }\end{array}$ & Y & GBD, DDW & Y & Y \\
\hline Bowie, 1997 [22] & Incidence & $\begin{array}{l}\text { Own developed } \\
\text { groups }\end{array}$ & n.a. & Y & Y & N & N & $\begin{array}{l}\text { Standard West } \\
25 \text { (females) and } \\
26 \text { (males) }\end{array}$ & N & GBD & N & N \\
\hline $\begin{array}{l}\text { Bradshaw, } \\
2003 \text { [14] }\end{array}$ & Incidence & $\begin{array}{l}\text { Adapted GBD } \\
\text { list (removed } \\
\text { causes due to } \\
\text { little relevance) }\end{array}$ & Y & Y & Y & Y & $\mathrm{N}$ & $\begin{array}{l}\text { Standard West } \\
25 \text { (males) and } \\
26 \text { (females) }\end{array}$ & Y & $\begin{array}{l}\text { GBD1990, } \\
\text { GBD2000, } \\
\text { Audw, DDW }\end{array}$ & Y & N \\
\hline $\begin{array}{l}\text { Bundhamcharoen, } \\
2002 \text { [16] }\end{array}$ & Incidence & GBD list I, II, III & Y & Y & Y & Y & N & Standard West 26 & Y & GBD, DDW & Y & Y \\
\hline $\begin{array}{l}\text { Chapman, } \\
2006 \text { [41] }\end{array}$ & Incidence & GBD list I, II, III & Y & Y & Y & Y & $N$ & Standard West 26 & Y & GBD, Zimb & $N$ & n.a. \\
\hline $\begin{array}{l}\text { Dodhia, } \\
2008 \text { [50] }\end{array}$ & Incidence & $\begin{array}{l}\text { Adapted GBD } \\
\text { list (removed } \\
\text { causes and } \\
\text { added own } \\
\text { groups) }\end{array}$ & Y & Y & $N$ & Y & $N$ & Standard West 26 & N & GBD & $N$ & $N$ \\
\hline $\begin{array}{l}\text { Hyder, } \\
2000 \text { [40] }\end{array}$ & Incidence & $\begin{array}{l}\text { Adapted GBD } \\
\text { list (removed } \\
\text { causes and } \\
\text { added own } \\
\text { groups) }\end{array}$ & n.a. & Y & N & Y & $N$ & $\begin{array}{l}\text { Standard West } \\
25 \text { (females) and } \\
26 \text { (males) }\end{array}$ & N & GBD & $N$ & $\mathrm{~N}$ \\
\hline $\begin{array}{l}\text { Innove Solutions, } \\
1998 \text { [51] }\end{array}$ & Incidence & $\begin{array}{l}\text { Adapted GBD } \\
\text { list (removed } \\
\text { causes and } \\
\text { added own } \\
\text { groups) }\end{array}$ & $\mathrm{N}$ & $N$ & N & N & N & Standard West 26 & Y & GBD, DDW & $\mathrm{N}$ & n.a. \\
\hline $\begin{array}{l}\text { Jankovic, } \\
2006 \text { [52] }\end{array}$ & Incidence & $\begin{array}{l}\text { Adapted GBD } \\
\text { list (removed } \\
\text { causes due } \\
\text { to little } \\
\text { relevance) }\end{array}$ & Y & Y & Y & Y & $N$ & Standard West 26 & n.a. & $\begin{array}{l}\text { GBD1990, } \\
\text { GBD2000, } \\
\text { DDW }\end{array}$ & Y & $N$ \\
\hline Laaser, 2007 [38] & $\begin{array}{l}\text { Incidence and } \\
\text { prevalence }\end{array}$ & GBD list I, II, III & n.a. & Y & Y & Y & $N$ & $\begin{array}{l}\text { Standard West } \\
25 \text { (females) and } \\
26 \text { (males) }\end{array}$ & Y & $\begin{array}{l}\text { GBD1996, } \\
\text { GBD2000 }\end{array}$ & n.a. & $\mathrm{N}$ \\
\hline
\end{tabular}


Table 2 Methods used to calculate YLL and YLD in general burden of disease studies (Continued)

\begin{tabular}{|c|c|c|c|c|c|c|c|c|c|c|c|c|}
\hline Lai, 2009 [27] & Prevalence & GBD list I, II, III & Y & $\mathrm{N}$ & $\mathrm{N}$ & N & $N$ & $\begin{array}{l}\text { Standard life tables } \\
\text { of Estonia }\end{array}$ & N & Estdw & Y & N \\
\hline Lopez, 2006 [9] & Incidence & GBD list I, II, III & n.a. & Y & $N$ & Y & Y & $\begin{array}{l}\text { Standard West } \\
25 \text { (females) and } \\
26 \text { (males) }\end{array}$ & Y & GBD & n.a. & N \\
\hline $\begin{array}{l}\text { Mathers, } \\
2001[28]\end{array}$ & Incidence & $\begin{array}{l}\text { Adapted GBD } \\
\text { list (added own } \\
\text { groups) }\end{array}$ & Y & Y & $\mathrm{N}$ & Y & Y & $\begin{array}{l}\text { Australian standard } \\
\text { life tables }\end{array}$ & Y & DDW, GBD & Y & Y \\
\hline Melse, 2000 [21] & Prevalence & $\begin{array}{l}\text { Own developed } \\
\text { groups }\end{array}$ & n.a. & $\mathrm{N}$ & $N$ & Y & $N$ & $\begin{array}{l}\text { Dutch life tables } \\
\text { for } 1994\end{array}$ & Y & DDW & Y & N \\
\hline $\begin{array}{l}\text { Michaud, } \\
2006 \text { [35] }\end{array}$ & Incidence & $\begin{array}{l}\text { Adapted GBD } \\
\text { list (removed } \\
\text { causes and } \\
\text { added own } \\
\text { groups) }\end{array}$ & Y & Y & Y & Y & $\mathrm{N}$ & $\begin{array}{l}\text { Standard West } \\
\text { level } 25 \text { and } 26\end{array}$ & Y & GBD & Y & N \\
\hline $\begin{array}{l}\text { Murray, } \\
1997 \text { [10] }\end{array}$ & Incidence & GBD list I, II, III & n.a. & Y & Y & Y & Y & $\begin{array}{l}\text { Standard West } \\
\text { level } 25 \text { and } 26\end{array}$ & Y & GBD & n.a. & N \\
\hline $\begin{array}{l}\text { Naghavil, } \\
2009 \text { [31] }\end{array}$ & Incidence & $\begin{array}{l}\text { Adapted GBD } \\
\text { list (removed } \\
\text { causes and } \\
\text { added own } \\
\text { groups) }\end{array}$ & Y & Y & Y & N & $N$ & Iran life expectancy & Y & GBD, DDW & n.a. & Y \\
\hline Phua, 2009 [39] & Incidence & $\begin{array}{l}\text { Adapted GBD } \\
\text { list (removed } \\
\text { causes and } \\
\text { added own } \\
\text { groups) }\end{array}$ & Y & Y & $N$ & N & $\mathrm{N}$ & $\begin{array}{l}\text { Standard West } \\
25 \text { (females) and } \\
26 \text { (males) }\end{array}$ & Y & $\begin{array}{l}\text { GBD, DDW, } \\
\text { Audw }\end{array}$ & Y & N \\
\hline Pike, 2002 [30] & Incidence & $\begin{array}{l}\text { Adapted GBD } \\
\text { list (removed } \\
\text { causes and } \\
\text { added own } \\
\text { groups) }\end{array}$ & n.a. & Y & $N$ & N & $\mathrm{N}$ & $\begin{array}{l}\text { Australian standard } \\
\text { life tables }\end{array}$ & n.a. & GBD, DDW & Y & Y \\
\hline $\begin{array}{l}\text { SA Department } \\
\text { of Health } \\
\text { Australia, } \\
2005[12]\end{array}$ & Incidence & $\begin{array}{l}\text { Adapted GBD } \\
\text { list (added own } \\
\text { groups) }\end{array}$ & Y & Y & Y & Y & Y & $\begin{array}{l}\text { Australian standard } \\
\text { life tables }\end{array}$ & Y & GBD, DDW & Y & Y \\
\hline $\begin{array}{l}\text { Somerford, } \\
2004 \text { [29] }\end{array}$ & Incidence & GBD list I, II, III & N & Y & Y & Y & Y & $\begin{array}{l}\text { Australian standard } \\
\text { life tables }\end{array}$ & Y & N, YLL:YLD & $\mathrm{N}$ & N \\
\hline $\begin{array}{l}\text { Stevens, } \\
2008[26]\end{array}$ & Incidence & GBD list I, II, III & n.a. & Y & Y & N & Y & $\begin{array}{l}\text { Standard life tables } \\
\text { of Mexico }\end{array}$ & Y & GBD & $\mathrm{N}$ & N \\
\hline Tobias, 2001 [20] & Incidence & $\begin{array}{l}\text { Own developed } \\
\text { groups }\end{array}$ & Y & Y & Y & Y & Y & $\begin{array}{l}\text { Standard West } \\
25 \text { (females) and } \\
26 \text { (males) }\end{array}$ & n.a. & $\begin{array}{l}\text { DDW, GBD, } \\
\text { Audw, } \\
\text { EQ5Ddw }\end{array}$ & Y & Y \\
\hline Ünüvar, 2006 [15] & Incidence & GBD list I, II, III & Y & Y & Y & Y & Y & Standard West & Y & GBD, DDW & Y & N \\
\hline
\end{tabular}

(1)


Table 2 Methods used to calculate YLL and YLD in general burden of disease studies (Continued)

\begin{tabular}{|c|c|c|c|c|c|c|c|c|c|c|c|c|}
\hline $\begin{array}{l}\text { Victorian } \\
\text { BoD study, } \\
2005 \text { [19] }\end{array}$ & Incidence & $\begin{array}{l}\text { Adapted GBD } \\
\text { list (removed } \\
\text { causes and } \\
\text { added own } \\
\text { groups) }\end{array}$ & Y & Y & $\mathrm{N}$ & Y & Y & $\begin{array}{l}\text { Standard West } \\
25 \text { (females) and } \\
26 \text { (males) }\end{array}$ & Y & $\begin{array}{l}\text { DDW, GBD, } \\
\text { EQ5D, } \\
\text { Audw }\end{array}$ & Y & Y \\
\hline $\begin{array}{l}\text { World Health } \\
\text { Organization, } \\
2008 \text { [8] }\end{array}$ & Incidence & GBD list I, II, III & Y & Y & Y & Y & Y & $\begin{array}{l}\text { Standard West } \\
\text { level } 25 \text { and } 26\end{array}$ & Y & GBD & N & N \\
\hline $\begin{array}{l}\text { World Health } \\
\text { Organization, } \\
2009 \text { [11] }\end{array}$ & Incidence & GBD list I, II, III & Y & Y & Y & Y & Y & $\begin{array}{l}\text { Standard West } \\
\text { level } 25 \text { and } 26\end{array}$ & Y & GBD & N & N \\
\hline Yoon, 2007 [24] & n.a. & $\begin{array}{l}\text { Adapted GBD } \\
\text { list (removed } \\
\text { causes and } \\
\text { added own } \\
\text { groups) }\end{array}$ & Y & n.a. & n.a. & Y & N & Standard West & Y & KORdw & Y & N \\
\hline Yusoff, 2004 [17] & Incidence & GBD list I, II, III & Y & Y & N & $N$ & N & $\begin{array}{l}\text { Standard West } \\
25 \text { (females) and } \\
26 \text { (males) }\end{array}$ & Y & GBD, DDW & Y & N \\
\hline Zhao, 2004 [18] & Incidence & GBD list I, II, III & n.a. & n.a. & n.a. & $N$ & N & $\begin{array}{l}\text { Australian standard } \\
\text { life tables }\end{array}$ & Y & DDW, GBD & n.a. & N \\
\hline Zhou, 2011 [36] & Prevalence & $\begin{array}{l}\text { Adapted GBD } \\
\text { list (removed } \\
\text { causes) }\end{array}$ & n.a. & Y & n.a. & $\mathrm{N}$ & N & Standard West & Y & N, YLL:YLD & n.a. & N \\
\hline
\end{tabular}


described by sensitivity analysis in 21 studies (Table 2). These studies test whether plausible changes in values of the main variables affect the results of the analysis [1]. Most studies showed how the results of their study varied when a discount rate changes, and some studies also examined the influence of the use of age-weighting, the effect of uncertainty in the disability weights, and/or uncertainty in the incidence data.

\section{Risk factor analysis}

A risk factor is an attribute or exposure that is causally associated with an increased probability of a disease or injury [1]. Regarding the causal attribution of the burden of disease, one can either attribute it to a single cause (categorical attribution) or to a group of causes (counterfactual attribution). The latter can be analyzed using counterfactual analysis. With counterfactual analysis, the current or future disease burden is compared with the burden of disease that would be expected under an alternative hypothetical scenario, the counterfactual scenario, to estimate the effects of disease(s) or risk factor(s) [37].

Twelve studies performed risk factor analyses $[8-13,15,19,20,26,28,29]$. Risk factors that were analyzed were related to the effect of lifestyle factors (such as tobacco smoking, physical inactivity, alcohol consumption, diet, unsafe sex, and intimate partner violence), physiological states (such as obesity, high blood pressure, and high cholesterol) and also societal conditions (such as occupational exposures and air pollution) on the burden of disease.

\section{Comparison of disability-adjusted life year outcomes for specific disease and injury groups}

The total burden due to diseases and injuries varies enormously between the included studies. The highest burden of disease was found in Pakistan (45,600 DALYs per 100,000) and Zimbabwe (41,900 DALYs per 100,000). The lowest burden of disease was estimated in Queensland $(10,700$ DALYs per 100,000$)$ and Singapore (10,400 DALYs per 100,000) (Table 3). The differences in total DALYs between countries can partly be explained by differences in exposure to risk factors. For example, almost half of the total burden of disease in Zimbabwe is due to HIV and diarrhea. These diseases are rare in developed countries. In most developed countries the highest burden is caused by cardiovascular diseases, followed by road traffic injuries and depression.

Comparison with the GBD DALY outcomes by country revealed that DALY estimates were of similar magnitude in some studies (e.g., for Syria [38], USA [35], Singapore [39], and Turkey [15]). Other studies reported DALY estimates that were two times higher $[27,40]$ or two times lower [41] than the GBD study (Table 3).
Notably, four of the Australian burden of disease studies found comparable total DALY outcomes (between 13,220 and 13,700) $[12,13,19,28]$, where the GBD reported 9,894 as total DALYs for Australia.

\section{Discussion}

We systematically reviewed 31 general burden of disease studies using the DALY approach and performed a quality assessment of the methodology used. We found that studies generally followed the GBD approach, but that large differences exist in methodology. Most studies used the incidence-based approach (80\%), and almost all studies classified disease and injury groups as defined by the GBD. Half of the studies used age-weighting, whereas $80 \%$ of the studies used discounting.

As all systematic reviews, our study has some limitations. Reviewing the literature in the field of "burden of disease" studies was complicated by a wide variety of terminology for burden of disease. Consequently, some relevant publications may have been missed. To enhance the identification of relevant burden of disease studies we have used a variety of literature databases and keywords were matched to database-specific indexing terms. Furthermore, this review is limited to the English language. Therefore, relevant studies in other languages (e.g., Spanish $[42,43])$ are excluded. In the databases that were reviewed, we found a limited number of studies that included all diseases and injuries. An explanation for this finding may be that the use of the DALY is controversial and accompanied by theoretical concerns [44]. Practical concerns, such as lack of resources and available data sources and/or expertise, may also be a reason for researchers' apprehension to perform multiple-cause burden of disease studies.

\section{Quality of the data and were there any data gaps}

The main issue in burden of disease studies is access to complete, consistent, and comparable epidemiological data. Summary measures of population health, such as the DALY, are only as good as the weakest link in the chain, which is the epidemiological evidence [45]. Most studies derived numbers of incident cases directly from disease registers, routine databases, or epidemiological studies. Furthermore, some studies used a combination of incidence and prevalence-based data because, for most conditions, only prevalence data were available. The calculation of mortality burden is straightforward, and the precision of the estimates of YLL depends almost entirely on the quality of data on underlying causes of death. Although great improvements in reporting, coding, and classification of mortality have been made, significant challenges remain. The infrastructure for mortality and health databases varies considerably around the world [1,46]. Developed regions have 
Table 3 DALY outcomes for specific disease and injury groups (per 100,000 persons)

\begin{tabular}{|c|c|c|c|c|c|c|c|c|}
\hline \multirow[t]{2}{*}{ Author (ref no.) } & \multirow[t]{2}{*}{ Country } & \multirow{2}{*}{$\begin{array}{l}\text { GBD study } \\
\text { all causes* }\end{array}$} & \multicolumn{6}{|c|}{ Study outcome } \\
\hline & & & All causes & $\begin{array}{l}\text { Cardiovascular } \\
\text { diseases }\end{array}$ & $\begin{array}{l}\text { Road traffic } \\
\text { injury }\end{array}$ & $\begin{array}{l}\text { Diabetes } \\
\text { Mellitus }\end{array}$ & Depression & HIV/AIDS \\
\hline Begg [13] & England & Australia & 9,894 & 13,240 & 2,380 & 930 & 720 & 1,760 \\
\hline Bowie [22] & England & 11,012 & n.a. & n.a. & n.a. & n.a. & n.a. & n.a. \\
\hline Bradshaw [14] & South Africa & 46,137 & 36,100 & n.a. & $\begin{array}{l}2,800 \\
\text { (all unintentional) }\end{array}$ & n.a. & n.a. & 10,210 \\
\hline Bundhamchareon [16] & Thailand & 20,216 & 13,710 & 380 & 893 & 624 & 342 & 1,900 \\
\hline Chapman [41] & Zimbabwe & 82,801 & 41,930 & 293 & 461 & n.a. & 2,350 & 20,380 \\
\hline Dodhia [50] & England & 11,012 & 13,400 & 2,300 & n.a. & n.a. & 3,220 & n.a. \\
\hline Hyder [40] & Pakistan & 26,693 & 45,630 & 2,420 & n.a. & 1,170 & n.a. & 109 \\
\hline Innove Solutions [51] & England & 11,012 & n.a. & n.a. & n.a. & n.a. & n.a. & n.a. \\
\hline Jankovic [52] & $\begin{array}{l}\text { Serbia /Serbia } \\
\text { Montenegro }\end{array}$ & 14,562 & n.a. & 1,800 & 1,800 & 3,000 & 8,800 & 100 \\
\hline Laaser [38] & Syria & 16,167 & 14,700 & 3,700 & 1,120 & 610 & 840 & n.a. \\
\hline Lai [27] & Estonia & 16,212 & 32,700 & 5,700 & n.a. & n.a. & n.a. & n.a. \\
\hline Lopez [9] & Global & & Vary by region & n.a. & n.a. & n.a. & n.a. & n.a. \\
\hline Mathers [25] & Australia & 9,894 & 13,700 & 2,400 & 300 & 410 & 510 & n.a. \\
\hline Melse [21] & Netherlands & 9,948 & 16,000 & 2,720 & 460 & 540 & 710 & 100 \\
\hline Michaud [35] & USA & 12,844 & 12,420 & 1,730 & 520 & n.a. & n.a. & 360 \\
\hline Murray [10] & Global & & Vary by region & n.a. & n.a. & n.a. & n.a. & n.a. \\
\hline Naghavi [31] & Iran & 19,432 & 21,570 & 1,840 & 1,960 & n.a. & 900 & n.a. \\
\hline Phua [39] & Singapore & 10,111 & 10,400 & 2,050 & $\begin{array}{l}360 \\
\text { (all unintentional) }\end{array}$ & 1,130 & 1,200 & n.a. \\
\hline Pike [30] & Queensland & 9,894 & 10,710 & 2,260 & 240 & 320 & 1,400 & 8 \\
\hline $\begin{array}{l}\text { SA Dep Health, } \\
2005 \text { [12] }\end{array}$ & Australia & 9,894 & 13,220 & 2,700 & 950 & 400 & 1,800 & n.a. \\
\hline Somerford [29] & Australia & 9,894 & n.a. & n.a. & n.a. & n.a. & n.a. & n.a. \\
\hline Stevens [26] & Mexico & 9,894 & 14,501 & 450 & 700 & 510 & 900 & n.a. \\
\hline Tobias [20] & New Zealand & 10,642 & 15,100 & 3,620 & 450 & 574 & 1,810 & n.a. \\
\hline Ünüvar [15] & Turkey & 16,307 & 14,790 & 2,070 & 355 & 280 & 590 & n.a. \\
\hline Victorian BOD [19] & Australia & 9,894 & 13,600 & 860 & 230 & 1,120 & 860 & 25 \\
\hline WHO, 2008 [8] & Global & & Vary by region & n.a. & n.a. & n.a. & n.a. & n.a. \\
\hline WHO, 2009 [11] & Global & & Vary by region & n.a. & n.a. & n.a. & n.a. & n.a. \\
\hline Yoon [24] & Korea & 22,128 & n.a. & 1,490 & n.a. & 990 & 1,140 & n.a. \\
\hline Yusoff [17] & Malaysia & 16,638 & n.a. & n.a. & n.a. & n.a. & n.a. & n.a. \\
\hline Zhao [18] & $\begin{array}{l}\text { Australia } \\
\text { (non-aboriginals) }\end{array}$ & 9,894 & 18,320 & 2,600 & $\begin{array}{l}2,580 \\
\text { (all unintentional) }\end{array}$ & 440 & 3,060 & n.a. \\
\hline Zhou, 2011 [36] & China & 15,750 & 12,270 & 1,200 & $\begin{array}{l}1,470 \\
\text { (all unintentional) }\end{array}$ & 90 & n.a. & n.a \\
\hline
\end{tabular}

* age-standardized DALY per 100,000 by country. Source: Global Burden of Disease study 2004. http://www.who.int/healthinfo/global_burden_disease/ estimates_country/en/index.html.

electronic databases that provide summary statistics through World Wide Web-based queries. Other countries maintain tabulated mortality statistics that are not integrated into a utilizable database, and many developing countries have paper-based systems with rates based on projections and estimates rather than actual counts [46]. The data challenges that result from disparities in the level of health infrastructure yield rates that can be difficult to compare. Furthermore, differences in death certification systems, methods of data collection, 
and definitions of variables severely challenge international comparisons.

\section{Methodological choices}

The calculation of the morbidity component of the burden of disease, expressed in YLD, requires extensive epidemiological modelling and is often based on a diverse range of data sources, literature research, and/or expert opinion. The resulting YLD estimates depend highly on the specific model being applied and the type of data underlying this model.

Most studies used the GBD 1996 disability weights, in many cases supplemented by DDW. The GBD disability weights cover a wider range of conditions than covered by the DDWs, but are generally less specific in terms of the disease and sequelae categories to which they refer. The set of DDW covers a more restricted range of conditions compared to the GBD 1996 disability weights, but it differentiates more finely between condition stages and severities, thus allowing more detailed disease models in estimating the YLD than is possible with the GBD weights [47]. For example, DDWs are often used for HIV/AIDS and organ disorders. For the GBD 2010 Study, new disability weights are being derived [6].

In the original GBD study, discounting and ageweighting are applied. Both age-weighting and discounting have been disputed. The use of age-weighting is discussed, since lost years of healthy life are assumed to be of equal value regardless of the age at loss, the absence of empirical foundation and validation, and because the age weights do not convey actual social values as this practice is controversial $[3,48]$. Discounting has been disputed because its application results in a lower efficiency of prevention programs, whereas not discounting, or the use of a low discount rate - lower than the discount rate used for the costs - favors preventive measures due to benefit in the far future [32]. This discussion is reflected in different choices to use discounting and age-weighting between studies.

To measure the gap between actual population health and an ideal, most studies used the global standard life expectancy (West Level 26 and 25 life tables) as used by the GBD study. These life tables contain the relevant expectancies for each age and sex grouping. However, they have the disadvantage that they are abridged period life tables, chiefly using five-year age groupings and an upper-age category of $85+$ [28]. The use of cohort life expectancies with more complete underlying population data and more complex methods, as done for two Australian studies [12,28], resulted in more accurate and slightly different life expectancy measures. The use of the global standard life expectancy figures is recommended to enlarge the comparability with GBD study outcomes, but a sensitivity analysis using more detailed country-specific life expectancies is recommended.

\section{Comparison of DALY outcomes}

The sensitivity of DALYs, defined by the relative contributions of "true" and "error" variation, is assumed to be low. Potential sources of true variation include differences in the size and structure of populations, real differences in disease epidemiology between populations or over time, and differences in disability weights. Error variation may originate from the use of different methodologies (e.g., for discounting and age-weighting, disability weights) and from low-quality mortality and morbidity data. The detection of true variation is the focus of interest when estimating the burden of disease in DALYs. However, error may limit the power to detect true differences between populations [49]. Therefore, at the moment burden of disease studies are not comparable, nor are disease rankings as these are affected by methodological variation as well.

\section{Conclusions and recommendations}

Burden of disease analyses provide a unique perspective on health, one that integrates fatal and nonfatal outcomes, yet also allows the two classes of outcomes to be examined separately. Furthermore, burden of disease studies may provide a valuable insight into the scope for further health gains on the global or country level. This information will assist in taking up the future challenges posed by an aging population, by changes in disease and risk factor patterns, and by the increasing costs of health services [28]. Linking burden of disease analyses to cost effectiveness studies of interventions for major health problems will allow these interventions to be judged both in terms of cost effectiveness, and their relative impacts in reducing the burden of disease and ill health at the population level. Furthermore, burden of disease studies may shed light on crucial data gaps and facilitate priority setting in research.

However, large differences in used methodology exist between general burden of disease studies. Because of the methodological variation between studies it is difficult to assess whether differences in DALY estimates between the studies are due to actual differences in population health or whether these are the result of methodological choices. Overcoming this methodological rigor between burden of disease studies using the DALY approach is a critical priority for advancing burden of disease studies. Harmonization of the methodology used and high-quality data can enlarge the detection of true variation in DALY outcomes between populations or over time. 
Furthermore, overcoming this limitation in methodological rigor is particularly important in view of the imminent launch of the GBD 2010 Study, which is expected to result in a new impulse for the performance of burden of disease studies. It is a challenge for the GBD to develop more detailed harmonization procedures and clear guidelines to increase methodological improvements and enhanced comparability of general burden of disease studies.

\section{Competing interest}

The authors declare that they have no competing interest.

\section{Authors' contributions}

$\mathrm{SP}$ and $\mathrm{JH}$ carried out the search, selected included papers, independently critically appraised the selected papers, and developed the evidence tables. SP wrote the initial draft of the paper. AH was reviewer for included critically appraised papers, and contributed to the writing of the paper. $\mathrm{JH}, \mathrm{CS}$, and $\mathrm{AH}$ contributed substantially to the interpretation of study findings and writing the paper. All authors read and approved the final manuscript.

\section{Acknowledgement}

The work was supported by the World Health Organization. The work was carried out within the framework of Foodborne Disease Burden Epidemiology Reference Group (FERG). All authors read and approved the final manuscript.

\section{Author details}

'Department of Public Health, Erasmus MC, Rotterdam, Netherlands. ${ }^{2}$ Division of Information, Evidence, Research and Innovation, WHO Regional Office for Europe, Copenhagen, Denmark. ${ }^{3}$ National Institute for Public Health and the Environment, Laboratory for Zoonoses and Environmental Microbiology, Bilthoven, Netherlands. ${ }^{4}$ University Utrecht, Institute for Risk Assessment Sciences, Utrecht, Netherlands.

Received: 2 April 2012 Accepted: 15 October 2012

Published: 1 November 2012

\section{References}

1. Murray CJL, Lopez AD: The global burden of disease: A comprehensive assessment of mortality and disability from diseases, injuries and risk factors in 1990 and projected to 2020. Cambridge: Harvard University Press; 1996.

2. Worldbank: World Development Report 1993: Investing in Health. New York: Oxford University Press; 1993

3. Anand S, Hanson K: Disability-adjusted life years: a critical review. J Health Econ 1997, 16:685-702.

4. Barendregt JJ, Bonneux L, Van der Maas PJ: DALYs: The age-weights on balance. Bull World Health Organ 1996, 74:439-443.

5. Global Burden of Disease (GBD) 2010 study. http://www.who.int/healthinfo/ global_burden_disease/GBD_2005_study/en/index.html.

6. Salomon JA: New disability weights for the global burden of disease. Bull World Health Organ 2010, 88:879.

7. Wielders CC, van Lier EA, Van't Klooster TM, van Gageldonk-Lafeber AB, van den Wijngaard CC, Haagsma JA, Donker GA, Meijer A, van der Hoek W, Lugnér AK, et al: The burden of 2009 pandemic influenza $A(H 1 N 1)$ in the Netherlands. Eur J Public Health 2010, in press.

8. The global burden of disease: 2004 update. Geneva: World Health Organization; 2008

9. Lopez AD, Mathers CD, Ezzati M, Jamison DT, Murray CJ: Global and regional burden of disease and risk factors, 2001: systematic analysis of population health data. Lancet 2006, 367:1747-1757.

10. Murray CJ, Lopez AD: Alternative projections of mortality and disability by cause 1990-2020: global burden of disease study. Lancet 1997 349:1498-1504.

11. Global health risks: mortality and burden of disease attributable to selected major risks. Geneva: World Health Organization; 2009

12. SE Department of Health (DoH): Population health in South Australia: burden of disease and injury estimates, 1999-2001. Adelaide: Department of Health; 2005.
13. Begg SJ, Vos T, Barker B, Stanley L, Lopez AD: Burden of disease and injury in Australia in the new millennium: measuring health loss from diseases, injuries and risk factors. Med J Aust 2008, 188:36-40.

14. Bradshaw D, Groenewald P, Laubscher R, Nannan N, Nojilana B, Norman R, Pieterse D, Schneider M: Initial Burden of Disease Estimates for South Africa, 2000. Cape Town: South African Medical Research Council; 2003.

15. Ünüvar N, Mollahaliloglu S, Yardim N: Turkey Burden of Disease Study. Ankara: Ministry of Health; 2004.

16. Bundhamchareon K, Teerawattananon Y, Vos T, Begg S: Burden of disease and injuries in Thailand. Nonthaburi: Ministry of Public Health; 2002.

17. Yusoff AF, Mustafa AN, Kaur GK, Omar MA, Vos T, Rao VPC, Begg S: Malaysian Burden of Disease and Injury Study. In Forum 9. Mumbai, India: 2005:1-24.

18. Zhao Y, Guthridge S, Magnus A, Vos T: Burden of disease and injury in Aboriginal and non-Aboriginal populations in the Northern Territory. Med J Aust 2004, 180:498-502.

19. Victorian Burden of Disease Study: Mortality and morbidity in 2001. Melbourne: Victorian Government Department of Human Services; 2005

20. Tobias M: The Burden of Disease and Injury in New Zealand. Wellington: Ministry of Health; 2001.

21. Melse JM, Essink-Bot ML, Kramers PG, Hoeymans N: A national burden of disease calculation: Dutch disability-adjusted life-years. Dutch burden of disease group. Am J Public Health 2000, 90:1241-1247.

22. Bowie C, Beck S, Bevan G, Raftery J, Silverton F, Stevens A: Estimating the burden of disease in an English region. J Public Health Med 1997, 19:87-92.

23. Murray CJ: Quantifying the burden of disease: the technical basis for disability-adjusted life years. Bull World Health Organ 1994, 72:429-445.

24. Yoon SJ, Bae SC, Lee SI, Chang H, Jo HS, Sung JH, Park JH, Lee JY, Shin Y: Measuring the burden of disease in Korea. J Korean Med Sci 2007, 22:518-523.

25. Mathers CD, Vos T, Lopez AD, Salomon JA, Ezzati M: National Burden of Diseases Studies: A Practical Guide. Edition 2.0. Geneva: WHO Global Program on Evidence for Health Policy; 2001

26. Stevens G, Dias RH, Thomas KJ, Rivera JA, Carvalho N, Barquera S, Hill K, Ezzati M: Characterizing the epidemiological transition in Mexico: national and subnational burden of diseases, injuries, and risk factors. PLoS Med 2008, 5:e125.

27. Lai T, Habicht J, Kiivet RA: Measuring burden of disease in Estonia to support public health policy. Eur J Public Health 2009, 19:541-547.

28. Mathers CD, Vos ET, Stevenson CE, Begg SJ: The burden of disease and injury in Australia. Bull World Health Organ 2001, 79:1076-1084.

29. Somerford P, Katzenellenbogen J: Western Australian Burden of Disease Study: Disability-. Adjusted Life Years: Technical overview. Perth: Department of Health; 2004

30. Pike A, Baade P, Harper C, Muller S, Kennedy B: Quantifying the burden of disease and injury in Queensland 1996-1998. Brisbane, Australia: Queensland Government; 2002.

31. Naghavi M, Abolhassani F, Pourmalek F, Lakeh M, Jafari N, Vaseghi S, Mahdavi Hezaveh N, Kazemeini H: The burden of disease and injury in Iran 2003. Popul Health Metr 2009, 7:9.

32. Drummond M, O'Brien B, Stoddart GL, Torrance GW: Methods for the Economic Evaluation of Health Care Programmes. Oxford: Oxford Medical Publications; 1997

33. Murray CJ, Acharya AK: Understanding DALYs (disability-adjusted life years). J Health Econ 1997, 16:703-730.

34. Stouthard MEA, Essink-Bot ML, Bonsel GJ, DUTCH DISABILITY WEIGHTS (DDW) GROUP: Disability weights for diseases; a modified protocol and results for a Western European Region. Eur J Public Health 2000 10:24-30.

35. Michaud CM, McKenna MT, Begg S, Tomijima N, Majmudar M, Bulzacchelli MT, Ebrahim S, Ezzati M, Salomon JA, Kreiser JG, et al: The burden of disease and injury in the United States 1996. Popul Health Metr 2006, 4:11.

36. Zhou SC, Cai L, Wang J, Cui SG, Chai Y, Liu B, Wan CH: Measuring the burden of disease using disability-adjusted life years in Shilin County of Yunnan Province, China. Environ Health Prev Med 2011, 16:148-154.

37. Maldonado G, Greenland S: Estimating causal effects. Int J Epidemiol 2002 31:422-429.

38. Laaser U: The Burden of Diseases and Injury in Syria. Damascus: Ministry of Health; 2007.

39. Phua HP, Chua AV, Ma S, Heng D, Chew SK: Singapore's burden of disease and injury 2004. Singapore Med J 2009, 50:468-478. 
40. Hyder AA, Morrow RH: Applying burden of disease methods in developing countries: a case study from Pakistan. Am J Public Health 2000, 90:1235-1240

41. Chapman G, Hansen KS, Jelsma J, Ndhlovu C, Piotti B, Byskov J, Vos T: The burden of disease in Zimbabwe in 1997 as measured by disability-adjusted life years lost. Trop Med Int Health 2006, 11:660-671.

42. Gènova-Maleras R, Álvarez-Martín E, Catalá-López F, Fernández de Larrea-Baz N, M-G C: Burden of disease in the elderly population in Spain. Gac Sanit 2011, 25:47-50. Spanish.

43. Gómez Dantés HCM, Franco-Marina F, Bedregal P, Rodríguez García J, Espinoza A, Valdez Huarcaya W, América. LRRdlsCdEdOdSlp: Burden of disease in Latin America [Article in Spanish]. Salud Publica Mex 2011, 53:72-77.

44. Gouda HN, Powles JW: Why my disease is important: metrics of disease occurrence used in the introductory sections of papers in three leading general medical journals in 1993 and 2003. Popul Health Metr 2011, 9:14.

45. Polinder S, Haagsma JA, Lyons RA, Gabbe BJ, Ameratunga S, Cryer C, Derrett S, Harrison JE, Segui-Gomez M, van Beeck EF: Measuring the population burden of fatal and nonfatal injury. Epidemiol Rev 2012 34:17-31.

46. Chandran A, Hyder AA, Peek-Asa C: The global burden of unintentional injuries and an agenda for progress. Epidemiol Rev 2010, 32:110-120.

47. Essink-Bot ML, Bonsel GJ: How to derive disability weights. In Summary measures of population health: concepts, ethics, measurement and applications. Edited by Murray CJM, Salomon JA. Geneva: World Health Organization; 2002.

48. Johanneson M, Johansson PO: Is the valuation of QALY's gained independent of age? some empirical evidence. J Health Econ 1997, 16:589-599.

49. Essink-Bot ML, Pereira J, Packer C, Schwarzinger M, Burstrom K Cross-national comparability of burden of disease estimates: the European disability weights project. Bull World Health Organ 2002, 80:644-652.

50. Dodhia H, Phillips K: Measuring burden of disease in two inner London boroughs using disability adjusted life years. J Public Health (Oxf) 2008, 30:313-321.

51. West Pennine Burden of Disease Report 1998:: Centre for Health Care Development, West Pennine Health Authority and Living Media Ltd.

52. Jankovic S, Vlajinac H, Bjegovic V, Marinkovic J, Sipetic-Grujicic S, Markovic-Denic L, Kocev N, Santric-Milicevic M, Terzic-Supic Z, Maksimovic $\mathrm{N}$, Laaser U: The burden of disease and injury in Serbia. Eur J Public Health 2007, 17:80-85.

53. Stouthard ME, Essink-Bot ML, Bonsel GJ: Disability weights for diseases. A modified protocol and results for a Western European region. Eur J Public Health 2000, 10:24-30.

doi:10.1186/1478-7954-10-21

Cite this article as: Polinder et al.: Systematic review of general burden of disease studies using disability-adjusted life years. Population Health Metrics 2012 10:21.

\section{Submit your next manuscript to BioMed Central and take full advantage of:}

- Convenient online submission

- Thorough peer review

- No space constraints or color figure charges

- Immediate publication on acceptance

- Inclusion in PubMed, CAS, Scopus and Google Scholar

- Research which is freely available for redistribution 